\title{
Solitons in the Chiral Equation
}

\author{
Edwin J. Beggs \\ University College, Swansea, Wales SA2 8PP, United Kingdom
}

\begin{abstract}
The purpose of this paper is to give a geometric description of the solitons in the principal chiral equation in $1+1$ dimensions in terms of Grassmannians, and a qualitative description of their behaviour in terms of Morse functions. Additionally it shows how a soliton can be "added" to an arbitrary solution of the chiral equation.
\end{abstract}

\section{Table of Contents}

Section 1: The Linear System for Inverse Scattering . . . . . . . . 132

Section 2: The Evolution of Single Simple Pole Solitons. . . . . . . 133

Section 3: The Morse Functions for Time-Space Evolution. . . . . . . 134

Section 4: Superposition of Solitons . . . . . . . . . . . . . . 136

Section 5: Multiple Pole Solitons . . . . . . . . . . . . . . . 136

Section 6: The Group Action . . . . . . . . . . . . . . . . 139

\section{Introduction}

This paper has two objectives:

1. To give a simple geometric description of the solitons in the principal chiral equation in $1+1$ dimensions, and a qualitative description of their behaviour.

2. To show how a soliton can be "added" to an arbitrary solution of the chiral equation, and to give a geometric meaning to this in the case of addition of two solitons.

Our method is to start from the linear system of the inverse scattering procedure, which is well known $[4,6]$, and to find meromorphic solutions to this linear system. In the case where these meromorphic functions have one simple pole, the time space evolution can be described in terms of a Morse flow on a finite dimensional Grassmannian. In physical terms, the Morse function can be thought of as generating the envelope of the soliton. From this comes an intuitive argument showing how this solution gives rise to a non-dispersive travelling wave. 
The general meromorphic case can be studied by a flow on an infinite dimensional Grassmannian, and in this form the group action given by superimposing solitons can be easily seen. A similar Grassmannian approach has been used previously on the KdV equation [8]. It should be noted that a treatment of the group representation associated to the Euclidean version of the ciral equation (i.e. harmonic maps) has recently appeared [9].

\section{Section 1. The Linear System}

The chiral equation is most easily given in light cone coordinates, i.e. $\eta=t+x$ and $\xi=t-x$ in more usual time-space coordinates. It is a wave equation for a function $\sigma$ taking values in a unitary group instead of a linear space, i.e. $\sigma: \mathbb{R}^{1,1} \rightarrow U_{m}$. The equation is

$$
\sigma^{-1} \sigma_{\eta \xi}=\frac{1}{2}\left(\sigma^{-1} \sigma_{\eta} \sigma^{-1} \sigma_{\xi}+\sigma^{-1} \sigma_{\xi} \sigma^{-1} \sigma_{\eta}\right) .
$$

If we define the antihermitian functions

$$
A=\sigma^{-1} \sigma_{\xi} \text { and } B=\sigma^{-1} \sigma_{\eta},
$$

then (1.1) can be rewritten as

$$
A_{\eta}=\frac{1}{2}[A, B] \text { and } B_{\xi}=-\frac{1}{2}[A, B],
$$

and it is in this form that we shall most often refer to the chiral equation. By integrating along light lines and using the contraction mapping theorem, it can be shown that a unique solution exists for all space-time if the values of $A$ and $B$ are initially specified on a spacelike line.

Now consider the following linear equations for a function $\Psi: \mathbb{R}^{1,1} \times \mathbb{C}^{*}$ $\rightarrow G L_{m}(\mathbb{C}):$

$$
\Psi(\xi, \eta, \lambda)_{\xi}=\frac{\lambda+1}{2} \Psi A(\xi, \eta), \quad \Psi(\xi, \eta, \lambda)_{\eta}=\frac{\lambda^{-1}+1}{2} \Psi B(\xi, \eta) .
$$

This linear system is overdetermined, and the compatibility condition derived from $\Psi_{\eta \xi}=\Psi_{\xi \eta}$ is precisely (1.3). Thus if a $\Psi$ satisfying (1.4) exists, it gives a solution to the chiral equation.

Conversely, if $A$ and $B$ are solutions to (1.3), we can define $\Psi$ to be the solution to (1.4). This must exist by invariant integration since the compatibility condition is satisfied. Note that $\Psi$ is not uniquely defined, since we can specify it to be an arbitrary analytic function of $\lambda$ at $t=x=0$.

The most obvious non-trivial solution of (1.4) is

$$
\Psi_{0}(\eta, \xi, \lambda)=\exp \left(\frac{\lambda+1}{2} J \xi+\frac{\lambda^{-1}+1}{2} K \eta\right)
$$

where $J$ and $K$ are commuting antihermitian matrices. Note that if $\lambda=1$, this is just the most general group homomorphism from $\left(\mathbb{R}^{2},+\right)$ to $U_{m}$. If we want to study solutions to (1.4) which tend to $\Psi_{0}$ near infinity, then we remove $\Psi_{0}$ by defining

$$
\phi=\Psi_{0}^{-1} \Psi
$$


and observe that $\phi$ obeys the equations

$$
\phi_{\xi}=\frac{\lambda+1}{2}(\phi A-J \phi) \text { and } \phi_{\eta}=\frac{\lambda^{-1}+1}{2}(\phi B-K \phi) .
$$

\section{Section 2. The Evolution of Single Simple Pole Solitons}

We are going to describe the solitons in terms of a real form of the group of $m \times m$ invertible matrices with enteries in the field of rational functions of $\lambda \in \mathbb{C}_{\infty}$ (the Riemann sphere). We are interested in those solutions $\phi$ of (1.7) which lie in the real subgroup consisting of functions which are unitary on $\mathbb{R}_{\infty}$ (call these the meromorphic unitary loops).

It is known that any element of the real group is a product of a unitary matrix and single simple pole factors of the form

$$
\phi(\lambda)=(1-P)+\frac{\lambda-\bar{\alpha}}{\lambda-\alpha} \frac{1+\alpha}{1+\bar{\alpha}} P,
$$

where $P$ is an Hermitian projection on $\mathbb{C}^{m}$ and $\alpha \in \mathbb{C}-\mathbb{R}$. This will be proved as part of a factorization result in Sect. 5. We now seek a solution of the form (2.1) to (1.7), where $\alpha$ is fixed and $P$ is a function of $\xi$ and $\eta$, with $A$ and $B$ being, so far, unknown.

If $\phi_{\xi}$ is to be finite at $\lambda=\infty$, and $\phi_{\eta}$ finite at $\lambda=0$, we must have

$$
A=\phi(\xi, \eta, \infty)^{-1} J \phi(\xi, \eta, \infty) \text { and } B=\phi(\xi, \eta, 0)^{-1} K \phi(\xi, \eta, 0) .
$$

Substituting these relations back into (1.7) gives

$$
\begin{gathered}
2 P_{\xi}=(1+\bar{\alpha}) P J-(1+\alpha) J P+(\alpha-\bar{\alpha}) P J P, \\
2 P_{\eta}=\left(1+\bar{\alpha}^{-1}\right) P K-\left(1+\alpha^{-1}\right) K P+\left(\alpha^{-1}-\bar{\alpha}^{-1}\right) P K P .
\end{gathered}
$$

To get a geometric idea of what (2.3) means, we use the following lemma:

Lemma 2.4. Let $\theta$ be a $C^{1}$ function: $\mathbb{R} \rightarrow G L_{m}(\mathbb{C}), V_{0}$ be a subspace of $\mathbb{C}^{m}$, and define $V(x)=\theta(x) V_{0}$. Then if $P(x)$ is the orthogonal projection to $V(x)$ in $\mathbb{C}^{m}, P$ obeys the following equation:

$$
P^{\prime}(x)=P\left(\theta^{\prime} \theta^{-1}\right)^{*}+\left(\theta^{\prime} \theta^{-1}\right) P-P\left(\left(\theta^{\prime} \theta^{-1}\right)^{*}+\left(\theta^{\prime} \theta^{-1}\right)\right) P .
$$

Proof. By definition of $P$,

$$
(1-P(x)) \theta(x)=0 \quad \text { on } V_{0} .
$$

Differentiate this and use the formula

$$
P^{\prime}=P^{\prime} P+P P^{\prime}=P^{\prime} P+\left(P^{\prime} P\right)^{*} \text {. }
$$

We can now see that the solution to $(2.3)$ is that $P(\xi, \eta)$ is the orthogonal projection to the subspace $V(\xi, \eta)$ of $\mathbb{C}^{m}$ defined by

$$
V(\xi, \eta)=\exp \left(-\frac{1+\alpha}{2} J \xi-\frac{1+\alpha^{-1}}{2} K \eta\right) V_{0}
$$


for some subspace $V_{0}$ of $\mathbb{C}^{m}$. It is more informative to expand the exponential in this formula in two parts: Here let $\alpha$ have real and imaginary parts $\alpha=a+i b$,

$$
V(\xi, \eta)=\exp \left(-\frac{1+\alpha}{2} J \xi-\frac{1+\frac{a}{|\alpha|^{2}}}{2} K \eta\right) \exp \left(-\frac{i b}{2} J \xi+\frac{i b}{2|\alpha|^{2}} K \eta\right) V_{0} .
$$

Thus if we define $Q(\xi, \eta)$ to be the orthogonal projection to

we see that

$$
\exp \left(-\frac{i b}{2} J \xi+\frac{i b}{2|\alpha|^{2}} K \eta\right) V_{0}
$$

$$
P(\xi, \eta)=\exp \left(-\frac{1+a}{2} J \xi-\frac{1+\frac{a}{|\alpha|^{2}}}{2} K \eta\right) Q \cdot \exp \left(\frac{1+a}{2} J \xi+\frac{1+\frac{a}{|\alpha|^{2}}}{2} K \eta\right) .
$$

Now $Q$ obeys the equations

$$
2 Q_{\xi}=-i b(J Q+Q J-2 Q J Q), \quad 2 Q_{\eta}=\frac{i b}{|\alpha|^{2}}(K Q+Q K-2 Q K Q) .
$$

In effect, we have split the soliton into an envelope $Q$ for the wave, and an oscillatory part. The point of doing this is that the space-time evolution of $Q$ can be described by Morse functions.

\section{Section 3. The Morse Functions}

Let the Grassmannian $\operatorname{Gr}(n, m)$ be the space of $n$-dimensional subspaces of $\mathbb{C}^{m}$. Gr is essentially a space of orthogonal projections, so its tangent space consists of Hermitian operators, and a Riemannian metric can be defined by

$$
\left\langle H_{1}, H_{2}\right\rangle=\operatorname{trace}\left(H_{1} H_{2}\right) \quad\left(H_{1}, H_{2} \in T \mathrm{Gr}\right) .
$$

If $Q(t)$ is the time evolution of a point on the Grassmannian under a Morse function $f$, then by definition

$$
Q^{\prime}(t)=\nabla f(Q(t))
$$

where the gradient $\nabla f$ is defined by

$$
\langle\nabla f(Q), H\rangle=f^{\prime}(Q ; H) \quad \forall H \in T_{Q}(\mathrm{Gr}) .
$$

In our case we wish to find a Morse function for the $\xi$ evolution of $Q$, and this would be required to satisfy

$$
\left\langle Q_{\xi}, H\right\rangle=f^{\prime}(Q ; H)=-\frac{i b}{2} \operatorname{trace}(J Q H+Q J H-2 Q J Q H) \quad \forall H \in T_{Q}(\mathrm{Gr}) .
$$

If we substitute $f(Q)=-\frac{i b}{2} \cdot \operatorname{trace}(J Q)$ into this equation, and remember that $H Q+Q H=H$ for $H$ in the tangent space at $Q$, the equation is satisfied. A similar 
result can be given for the $\eta$ evolution, but we will find it most convenient to present the result in the usual time and space coordinates,

$$
\begin{aligned}
& \text { The time Morse function } T(Q)=-\frac{i b}{2} \operatorname{trace}\left(\left(J-\frac{1}{|\alpha|^{2}} K\right) Q\right), \\
& \text { The space Morse function } X(Q)=-\frac{i b}{2} \operatorname{trace}\left(\left(J+\frac{1}{|\alpha|^{2}} K\right) Q\right) \text {. }
\end{aligned}
$$

For convenience we shall write $X(Q)$ as $\operatorname{trace}\left(F_{X} Q\right)$, and $T(Q)$ as $\operatorname{trace}\left(F_{T} Q\right)$. If there is a stationary observer at the origin $t=x=0$, he will see $Q(t, x=0)$ evolve according to the Morse function $T(Q)$, and since the Grassmannian is compact, $Q(t, 0)$ will tend to a limit $Q(t=\infty, 0)$ as $t \rightarrow \infty$ which is a critical point of $T(Q)$. The critical points are precisely those $Q$ for which $F_{T} Q+Q F_{T}-2 Q F_{T} Q=0$, that is those $Q$ which commute with $F_{T}$. Now if all the eigenvalues of $F_{T}$ are distinct, the critical points of $T(Q)$ are isolated. But $F_{X}$ commutes with $F_{T}$, so in this case a critical point of $F_{T}$ is also critical for $F_{X}$. Then for large $t$ the space evolution near $Q(t, 0)$ will become very slow. In fact our observer will see $Q$ tend to a constant limit as $t \rightarrow \infty$ uniformly on any bounded spatial neighbourhood $\{t\} \times\left[-x_{0}, x_{0}\right]$. As the exponentials in (2.7) commute with $Q(\infty, 0)$, we see that $P, A$, and $B$ also tend to constant limits. That is, the amplitude of the oscillatory part of $P$ becomes smaller as $Q$ tends to $Q(\infty, 0)$. By (2.2) the limit of $A$ is $J$, and the limit of $B$ is $K$. We describe this situation by saying that there is no velocity zero soliton.

However if $F_{T}$ has repeated eigenvalues, there is a possibility that $Q(\infty, 0)$ is not a critical point for $F_{X}$. In this case our stationary observer will see $Q$ tend to the Morse flow $x \mapsto Q(\infty, x)$ uniformly on bounded spatial sets as $t \rightarrow \infty$. This limiting $Q(\infty, x)$ will in turn tend to a constant as $x$ tends to $\pm \infty$, giving the characteristic soliton envelope.

We deal with a moving soliton by a Lorentz transformation, which is given in light cone coordinates by $\xi^{\prime}=u \xi, \eta^{\prime}=\eta / u$, where $u$ is a positive real number. (The positivity condition is to preserve the direction of time.) The velocity of an observer stationary in the new frame, with respect to the old frame, is now $\frac{1-u^{2}}{1+u^{2}}$, and the new space-time Morse functions are:

$$
\begin{aligned}
& T(Q, u)=-\frac{i b}{2} \operatorname{trace}\left(\left(\frac{1}{u} J-\frac{u}{|\alpha|^{2}} K\right) Q\right)=\operatorname{trace}\left(F_{T}(u) Q\right), \\
& X(Q, u)=-\frac{i b}{2} \operatorname{trace}\left(\left(\frac{1}{u} J+\frac{u}{|\alpha|^{2}} K\right) Q\right)=\operatorname{trace}\left(F_{X}(u) Q\right) .
\end{aligned}
$$

Then there can only be a soliton moving at velocity $\frac{1-u^{2}}{1+u^{2}}$ if the matrix $F_{T}(u)$ has repeated eigenvalues. If $J$ and $K$ have no repeated eigenvalues, there are no solitons moving at the speed of light. It is also possible to produce tachyons, that is solitons moving faster than light. In this case we have to reverse the roles of time and space, and observe that to have a tachyon moving at velocity $\frac{1+u^{2}}{1-u^{2}}$ we must have repeated eigenvalues for the matrix $F_{X}(u)$. If we wanted to set up a system 
which did not allow tachyons, all we would have to do is to insist that $J$ and $K$ could be simultaneously diagonalized so that they both had $i \times$ eigenvalues in strictly increasing order.

\section{Section 4. Superposition of Solitons}

We shall now describe a process which associates to each solution of the chiral equation another solution, in a manner which can be thought of as "addition of a soliton." Suppose that $C, D$ is such a solution, i.e.

$$
C_{\eta}=\frac{1}{2}[C, D] \text { and } D_{\xi}=-\frac{1}{2}[C, D] \text {. }
$$

Now look for a single simple pole unitary meromorphic solution $\varrho$ to the equation

$$
\varrho_{\xi}=\frac{\lambda+1}{2}(\varrho A-C \varrho) \text { and } \varrho_{\eta}=\frac{\lambda^{-1}+1}{2}(\varrho B-D \varrho) \text {. }
$$

By continuing in exactly the same manner as in the second section, we find that a solution to this problem with a pole at $\alpha$ can be written as

$$
\varrho(\lambda)=(1-P)+\frac{\lambda-\bar{\alpha}}{\lambda-\alpha} \frac{1+\alpha}{1+\bar{\alpha}} P,
$$

where $P$ is an orthogonal projection to the subspace $\theta(\xi, \eta) V_{0}$, and $\theta(\xi, \eta)$ obeys the equations

$$
\theta_{\xi} \theta^{-1}=-\frac{1}{2}(1+\alpha) C \text { and } \theta_{\eta} \theta^{-1}=-\frac{1}{2}\left(1+\alpha^{-1}\right) D
$$

The existence of such a $\theta$ can be demonstrated by solving the following invariant integration problem. Let $\theta(\xi, \eta)=r(\xi, \eta, 1)$, where $r(\xi, \eta, s)$ is the solution to

$$
\frac{\partial r}{\partial s} r^{-1}(\xi, \eta, s)=-\xi \frac{1+\alpha}{2} C(s \xi, s \eta)-\eta \frac{1+\alpha^{-1}}{2} D(s \xi, s \eta)
$$

for $s \in[0,1]$ and boundary condition $r(\xi, \eta, 0)=1$.

It should be noted that if $\mu(\xi, \eta, \lambda)$ is an extended solution fo: $C$ and $D$, that is if

$$
\mu_{\xi}=\frac{\lambda+1}{2}(\mu C-J \mu) \text { and } \mu_{\eta}=\frac{\lambda^{-1}+1}{2}(\mu D-K \mu),
$$

then we can define $\phi=\mu \varrho$, which will be a solution of (1.7). We say that $A, B$ is the solution obtained from $C, D$ by adding a soliton, and that $\phi$ is the extended solution associated to $A, B$.

\section{Section 5. Multiple Pole Solitons}

Here we introduce a Grassmannian $(5 r$, which we will show is in 1-1 correspondence with the set of all unitary meromorphic loops. Then the evolution of multiple pole solitons will be given by a flow on this Grassmannian.

Define $\mathfrak{U}$ to be the analytic functions from $\mathbb{C}^{*}$ to $\mathbb{C}$, and $\mathfrak{F}$ to be the meromorphic functions from $\mathbb{C}^{*}$ to $\mathbb{C}$ which have only finitely many poles. Then $\mathfrak{F}$ 
and $\mathfrak{F}^{m}$ are modules over the ring $\mathfrak{A}$. Also define a conjugation on functions by the formula $\bar{f}(\lambda)=\bar{f}(\bar{\lambda})$. There is a sesquilinear pairing: $\mathfrak{F}^{m} \times \mathfrak{F}^{m} \rightarrow \mathfrak{F}$ given by the formula

$$
\langle f, g\rangle(\lambda)=\sum_{i=1}^{m} \bar{f}_{i}(\lambda) g_{i}(\lambda)=\langle f(\bar{\lambda}), g(\lambda)\rangle_{\mathbb{C}^{m}}
$$

If $W$ is an $\mathfrak{A}$ module in $\mathfrak{F}^{m}$, define $W^{\perp}$ to be

$$
W^{\perp}=\left\{x \in \mathfrak{F}^{m}:\langle x, y\rangle \in \mathfrak{U} \forall y \in W\right\} .
$$

At last, the Grassmannian can be defined as

$$
\begin{aligned}
& \mathfrak{G r}=\left\{\mathfrak{A} \text { modules } W \subseteq \mathfrak{F}^{m}: W^{\perp}=W \text { and } \exists \text { polynomials } p, q\right. \\
&\text { so that } \left.p \mathfrak{A}^{m} \subseteq W \subseteq \frac{1}{q} \mathfrak{A}^{m}\right\}
\end{aligned}
$$

and the unitary meromorphic loops normalized at -1 , as

$$
G=\left\{\gamma \in G L_{m}\left(\operatorname{Mero}\left(\mathbb{C}_{\infty}, \mathbb{C}_{\infty}\right)\right): \bar{\gamma}^{T}=\gamma^{-1} \text { and } \gamma(-1)=1\right\} .
$$

The following proposition follows immediately from the definitions:

Proposition 5.5. There is a group action of $G$ on $\mathbf{G r}$, given by the formula

$$
(\gamma x)_{i}=\sum_{j} \gamma_{i j} x_{j} \text { for } x \in W \in \mathfrak{G r} .
$$

Proposition 5.6. The map: $G \rightarrow \mathfrak{G r}$ given by $\gamma \mapsto \gamma \mathfrak{A}^{m}$ is a 1-1 correspondence.

Proof of 1-1. Since we are dealing with a group action all we have to do is show that $\gamma \mathfrak{U}^{m}=\mathfrak{U}^{m}$ implies that $\gamma=1$. If $\gamma \in G$, then $\gamma$ is unitary on $\mathbb{R}^{*}$, so any poles must be on $\mathbb{C}^{*}-\mathbb{R}^{*}$. But if $\gamma$ had a pole in $\mathbb{C}^{*}$, then there would be a constant vector $a \in \mathbb{C}^{m}$ so that $\gamma a$ had a pole in $\mathbb{C}^{*}$, and this would mean that $\gamma \mathfrak{U} \nsubseteq \mathfrak{U}^{m}$. Therefore $\gamma$ has no poles, and is thus constant, and that constant is $\gamma(-1)=1$.

Proof of onto: If $W \in\left(\mathfrak{G r}\right.$, then $\frac{W}{W \cap \mathfrak{U}^{m}}$ is finite dimensional, and the proof is by induction on this dimension. The map given by multiplying by $\lambda$,

$$
\lambda \times: \frac{W}{W \cap \mathfrak{U}^{m}} \rightarrow \frac{W}{W \cap \mathfrak{A}^{m}}
$$

has at least one eigenvalue $\alpha$ if $W \nsubseteq \mathfrak{U}^{m}$. Then take a basis $\left[f_{1}\right], \ldots,\left[f_{r}\right]$ of $\operatorname{ker}(\lambda-\alpha) \times$, where $f_{i} \in W$, and note that $g_{i}=(\lambda-\alpha) f_{i}$ is in $W \cap \mathfrak{U}^{m}$. Now the vectors $g_{1}(\alpha), \ldots, g_{r}(\alpha)$ are linearly independent in $\mathbb{C}^{m}$, and we call $V$ the subspace of $\mathbb{C}^{m}$ which they span. Now let $P$ be the orthogonal projection to $V$, and define a simple meromorphic loop $\theta$ as

$$
\theta(\lambda)=(1-P)+\frac{\lambda-\alpha}{\lambda-\bar{\alpha}} \frac{1+\bar{\alpha}}{1+\alpha} P .
$$

Then there is an onto map

$$
[\theta \times]: \frac{W}{W \cap \mathfrak{U}^{m}} \rightarrow \frac{\theta W}{\theta W \cap \mathfrak{U}^{m}}
$$


which reduces dimension since $\left[f_{1}\right], \ldots,\left[f_{r}\right]$ are in the kernel. Then the process is repeated using $\theta W$ instead of $W$, until we obtain $\theta_{k} \ldots \theta_{1} \mathbf{W} \cong \mathfrak{A}^{m}$. On taking perpendiculars in this formula, we see that $W=\theta_{k}^{-1} \ldots \theta_{1}^{-1} \mathfrak{Q}^{m}$, thus proving the 1-1 correspondence between the Grassmannian and the unitary meromorphic loops. result:

In the course of the proof of the last proposition we also proved the following

Proposition 5.7. Any meromorphic unitary loop is a product of a unitary matrix and a number of single simple pole factors of the form

$$
\theta(\lambda)=(1-P)+\frac{\lambda-\bar{\alpha}}{\lambda-\alpha} \frac{1+\alpha}{1+\bar{\alpha}} P,
$$

where $P$ is a self-adjoint projection on $\mathbb{C}^{m}$. (Note the factorization is not unique in general.)

Now we are in a position to prove the main result:

Theorem 5.8. If $\phi(0,0)$ is a unitary meromorphic loop, there is a unique unitary meromorphic solution $\phi(\xi, \eta)$ to the equations

$$
\phi_{\xi}=\frac{\lambda+1}{2}(\phi A-J \phi) \text { and } \phi_{\eta}=\frac{\lambda^{-1}+1}{2}(\phi B-K \phi),
$$

and this $\phi$ is given by a flow on the Grassmannian defined by

$$
\phi(\xi, \eta) \cdot \mathfrak{A}^{m}=\exp \left(-\frac{\lambda+1}{2} J \xi-\frac{\lambda^{-1}+1}{2} K \eta\right) \phi(0,0) \cdot \mathfrak{A}^{m} .
$$

Proof. The existence result is proved by factorizing $\phi(0,0)$ into simple pole parts, and using the method of superimposing solitons discussed in the last section. The uniqueness will follow if we can show that $\phi(\xi, \eta)$ obeys the formula for the Grassmannian planes in the statement of the theorem. This will be proved for the $\xi$ evolution, and the proof for $\eta$ is of the same type. It is sufficient to show that

$$
\phi(0,0) \cdot \mathfrak{A}^{m} \leqq \exp \left(\frac{\lambda+1}{2} J \xi\right) \phi(\xi, 0) \cdot \mathfrak{A}^{m} .
$$

Start with an arbitrary $f(0) \in \mathfrak{A}^{m}$, and solve the following equation for $f(\xi)$;

$$
\frac{d}{d \xi}\left(\exp \left(\frac{\lambda+1}{2} J \xi\right) \phi(\xi, 0) f(\xi)\right)=0 .
$$

This will be true if $f_{\xi}=-\frac{\lambda+1}{2} A f$, which can be solved by invariant integration with $f(\xi) \in \mathfrak{A}^{m}$. But this proves that

$$
\phi(0,0) f(0) \in \exp \left(\frac{\lambda+1}{2} J \xi\right) \phi(\xi, 0) \cdot \mathfrak{A}^{m}
$$

for arbitrary $f(0) \in \mathfrak{U}^{m}$, as required. 


\section{Section 6. The Group Action}

Here we summarize the operation of soliton addition, as given in Sect. 4, specializing to the case of soliton solutions. It will be helpful to use a form of "Heisenberg" representation, that is the state space remains constant, and the interpretation varies as a function of space-time. It is also necessary to choose a preferred point in space-time, which we take to be the origin $t=x=0$.

The state space is taken to be infinite dimensional Grassmannian $\mathbf{G r}$, with the meromorphic unitary loop $\phi(0,0)$ corresponding to the state $\phi(0,0) \mathfrak{U}^{m} \in \mathfrak{G r}$. Then the unitary meromorphic loop valued interpretation function

is defined by

$$
\Phi: \mathbb{R}^{1,1} \times \mathfrak{G r} \rightarrow G
$$

$$
\Phi(\xi, \eta, W) \mathfrak{A}^{m}=\exp \left(-\frac{\lambda+1}{2} J \xi-\frac{\lambda^{-1}+1}{2} K \eta\right) W .
$$

In other words, if $\phi(\xi, \eta)$ is our usual soliton evolution beginning with $\phi(0,0)$, then

$$
\Phi\left(\xi, \eta, \phi(0,0) \mathfrak{A}^{m}\right)=\phi(\xi, \eta) .
$$

The advantage of writing the result of (5.8) this way is that we can describe the operation of adding the unitary meromorphic loop $\tau(0,0)$ to the existing soliton $\phi$ by the right action

$$
((\phi) \tau)(\xi, \eta)=\Phi\left(\xi, \eta, \phi(0,0) \tau(0,0) \mathfrak{A}^{m}\right) .
$$

Thus the operation of soliton addition described in Sect. 4 is just right multiplication of loops at the preferred point.

Acknowledgements. I would like to express my gratitude to Graeme Segal, for suggesting the problem and for much helpful advice. Also I would like to thank the Department of Education (Northern Ireland), and St. Catherine's and Christ Church colleges Oxford for their support. This work was written up whilst at Swansea, supported by the Science and Engineering Research Council.

\section{References}

1. Beggs, E.J.: D. Phil. thesis, Oxford

2. Dodd, R.K., Eilbeck, J.C., Gibbon, J.D., Morris, H.C.: Solitons and nonlinear wave equations. London: Academic Press 1982

3. Faddeev, L.D.: A Hamiltonian interpretation of the Inverse Scattering method. Topics in current physics, vol. 17 (Solitons), pp. 339-354. Berlin, Heidelberg, New York: Springer 1980

4. Faddeev, L.D., Takhtajan, L.A.: Hamiltonian methods in the theory of Solitons. Berlin, Heidelberg, New York: Springer 1987

5. Milnor, J.: Morse theory. Annals Maths. Stud. 51 (1963)

6. Novikov, S., Manakov, S.V., Pitaevskii, L.P., Zakharov, V.E.: Theory of solitons. Contemporary Soviet Mathematics, Consultants Bureau 1984

7. Pressley, A., Segal, G.B.: Loop groups. Oxford: Oxford University Press 1988

8. Segal, G.B., Wilson, G.: Loop groups and equations of KdV type. Pub. Math. I.H.E.S. 61, 5-65 (1985)

9. Uhlenbeck, K.: Harmonic maps into Lie Groups (classical solutions of the Chiral model). J. Diff. Geom. 30, 1-50 (1989)

Communicated by A. Jaffe

Received December 7, 1988 
